\title{
The Effect of Chronic Use of Proton Pump Inhibitors on Gastric Cancer: Should We Be Aware of It?
}

\author{
Christos Liatsos $^{\mathrm{a}}$ Theodore Rokkas $^{\mathrm{b}}$ \\ ${ }^{a}$ Department of Gastroenterology, 401 Army General Hospital of Athens, Athens, Greece; \\ ${ }^{b}$ Gastroenterology Clinic, Henry Dunant Hospital, Athens, Greece
}

To the Editor,

We read with great interest the recent review by Joo et al. [1], who concluded that the association between the use of chronic proton pump inhibitor (PPIs) and gastric cancer (GC) risk is still controversial and needs further evaluation. Although we generally agree with this statement, there are some areas that deserve comment. The authors based their results on some old studies as well as on a small recent metaanalysis with results that should be interpreted carefully due to the possibility of unadjusted crucial confounding factors such as Helicobacter pylori infection, a wellknown cancer-causing agent. It is worthwhile to mention 2 quite recent studies that try to clarify this issue. First, Cheung et al. [2], in a retrospective cohort populationbased study with a mean observation period of 7.5 years addressed the risk of GC development in $H$. pylori (+) patients after receiving eradication treatment. They found that the long-term use of PPIs increased the risk for GC, particularly for non-cardia cancer, and that the risk increased with longer duration of PPI use (hazard ratio [HR] 5.04, 95\% CI 1.23-20.61 for $\geq 1$ year of use; HR 6.65, 95\% CI $1.62-$ 27.26 for $\geq 2$ years of use and HR 8.34, 95\% CI 2.02-34.41 for $\geq 3$ years of use). They also found that daily use of PPIs was associated with 4.55 times increased risk compared to weekly use. So, they suggested that physicians should cautiously prescribe PPIs to $H$. pylori-infected individuals even after successful $H$. pylori eradication. Undoubtedly, results obtained may not be transferable to Western populations where GC risk is significantly lower. Second, Brusselaers et al. [3], in a population-based nationwide cohort study with 797,067 individuals on maintenance PPI treatment, showed that the standardized incidence ratios (SIRs) of GC were more than threefold higher (SIR = 3.38, 95\% CI 3.23-3.53). Moreover, the highest SIRs were found among participants exposed to indications with a known association with GC (H. pylori, peptic ulcer disease), whereas SIRs were also increased for indications without any such association. The authors emphasize that their single, although large study cannot determine causality. Nevertheless, the fact that long-term PPI use could be a potentially independent risk factor for GC cannot be dismissed.

It is well known that the long-term PPI treatment raises strong concerns in relation to digestive (e.g., hypergastrinemia induction, infectious [C. difficile in- fection, small intestinal bacteria overgrowth, spontaneous bacterial peritonitis in cirrhotic patients] and non-infectious [celiac disease] consequences, dysbiosis, electrolyte or nutrient absorption, idiosyncratic reactions) and extra-digestive tract effects (e.g., infections such as community-acquired pneumonia, risk of fractures, cardiovascular risk) [4]. Despite the paucity of robust data and randomized control trials in accordance with the aforementioned large observational studies, we believe that when the medical community analyzes the safety profiles of long-term PPIs' use it should insist upon the risk of being affected by GC. It is time for clinicians to minimize PPI over-prescription, especially to chronic users, and to follow evidence-based indications. Undoubtedly and in concordance with the suggestions of Joo et al. [1], large randomized control trials are needed to adequately assess any strong causality between PPI use and GC.

\section{Disclosure Statement}

The authors declare that there are no conflicts of interest of disclose.

\section{KARGER}

(C) 2018 S. Karger AG, Basel
Christos Liatsos, $\mathrm{MD}, \mathrm{PhD}$

Department of Gastroenterology, 401 Army General Hospital of Athens, Fellow of the European Board of Gastroenterology and Hepatology PO Box 833, GR-19009, Pikermi - Rafina (Greece)

E-Mail cliatsos@yahoo.com 


\section{References}

-1 Joo MK, Park JJ, Chun HJ: Additional benefits of routine drugs on gastrointestinal cancer: statins, metformin, and proton pump inhibitors. Dig Dis 2018;36:1-14.

-2 Cheung KS, Chan EW, Wong AYS, Chen L, Wong ICK, Leung WK: Long-term proton pump inhibitors and risk of gastric cancer de- velopment after treatment for Helicobacter pylori: a population-based study. Gut 2018; 67:28-35.

Brusselaers N, Wahlin K, Engstrand L, Lagergren J: Maintenance therapy with proton pump inhibitors and risk of gastric cancer: a nationwide population-based co- hort study in Sweden. BMJ Open 2017;7: e017739.

-4 Scarpignato C, Gatta L, Zullo A, Blandizzi C: Effective and safe proton pump inhibitor therapy in acid-related diseases - a position paper addressing benefits and potential harms of acid suppression. BMC Med 2016;14:179. 